\title{
Book Review—Diagnostic pathology: cardiovascular, 2nd edition
}

\author{
Cristina Basso ${ }^{1}$
}

Received: 25 September 2018 / Accepted: 27 September 2018 / Published online: 12 October 2018

(C) Springer-Verlag GmbH Germany, part of Springer Nature 2018

The second edition of this comprehensive textbook is an important contribution to the literature on cardiovascular pathology and a most welcome source book for practicing pathologists. It encompasses all aspects of cardiovascular diseases with a practical approach.

In fact, the book comprises nine sections, from endomyocardial biopsy, to explant and surgical heart and vascular resections, and to autopsy. The structure in sections reflects the routine daily practice in the pathology lab.

Each entity is briefly summarized with a "key facts" table, including terminology, etiopathogenesis, clinical aspects, macroscopic and microscopic key findings, and top differential diagnosis. These aspects are then addressed in more detail, where data are presented in a readable and concise format and supported by a few selected, updated references on the topic.

All chapters are well illustrated with top quality, color pictures like an atlas of cardiovascular diseases. Clinic pathologic correlations are included when necessary.
Of note, the book contains data not only on how to macroscopically and microscopically diagnose various cardiovascular conditions, but also provides important essential clinical information to interact more efficiently with cardiovascular surgeons and clinical and interventional cardiologists.

This book is recommended to general and forensic pathologists, pathologists in training, as well as the professed pathology experts.

Diagnostic Pathology: Cardiovascular, 2nd Edition Dylan V. Miller \& Monica P. Revelo

Print ISBN 9780323595605

$552 \mathrm{pp}$.

$£ 183.99$

https://www.uk.elsevierhealth.com/diagnostic-pathologycardiovascular-9780323595605.html

Cristina Basso

cristina.basso@unipd.it

1 Cardiovascular Pathology Unit, Department of Cardiac, Thoracic, Vascular Sciences and Public Health, University of Padua, Padua, Italy 